Applied Remote Sensing

Validation of the moderate-resolution imaging spectroradiometer land surface emissivity products over the Taklimakan Desert

Jie Cheng

Shunlin Liang

Lixin Dong

Baiyang Ren

Linpeng Shi 


\title{
Validation of the moderate-resolution imaging spectroradiometer land surface emissivity products over the Taklimakan Desert
}

\author{
Jie Cheng, ${ }^{\mathrm{a}, *}$ Shunlin Liang, ${ }^{\mathrm{a}, \mathrm{b}}$ Lixin Dong, ${ }^{\mathrm{c}}$ Baiyang Ren, ${ }^{\mathrm{a}}$ and \\ Linpeng Shi ${ }^{\mathrm{a}}$ \\ ${ }^{\text {a} B e i j i n g ~ N o r m a l ~ U n i v e r s i t y, ~ C o l l e g e ~ o f ~ G l o b a l ~ C h a n g e ~ a n d ~ E a r t h ~ S y s t e m ~ S c i e n c e, ~ S t a t e ~ K e y ~}$ \\ Laboratory of Remote Sensing Science, Beijing 100875, China \\ ${ }^{b}$ University of Maryland, Department of Geographical Science, College Park 20742, Maryland \\ ${ }^{\mathrm{c}}$ National Satellite Meteorological Center, Key Laboratory of Radiometric Calibration and \\ Validation for Environmental Satellites, Beijing 100081, China
}

\begin{abstract}
Land surface emissivity is a key parameter in estimating the land surface radiation budget. The validation of the moderate-resolution imaging spectroradiometer (MODIS) land surface emissivity with field measurements is rarely performed. In this study, a field measurement was performed over the central part of the Taklimakan Desert for the validation of the MODIS land surface emissivity products (MOD11B1) Version 4 (V4.1) and Version 5 (V5). The homogeneity of two validation sites was verified using the advanced spaceborne thermal emission and reflection radiometer (ASTER) land surface temperature and emissivity acquired closely before and after the overpass of MODIS. MOD11B1 V4.1 and V5 emissivity data for bands 29, 31, and 32 were compared to the emissivity calculated from the field measured emissivity spectra convolved with the filter function of the MODIS bands $29(8.52 \mu \mathrm{m}), 31(11.03 \mu \mathrm{m})$, and 32 $(12.04 \mu \mathrm{m})$. The comparison results indicate that the V4.1 emissivity data agree well with the field measurements, with mean absolute differences of 0.017 and 0.007 for site 1 and site 2, respectively, and the mean absolute differences of the V5 emissivity data were 0.034 and 0.033 for site 1 and site 2, respectively. The data version used must be considered when MOD11B1 is used in real applications, especially for time series analysis. (C) The Authors. Published by SPIE under a Creative Commons Attribution 3.0 Unported License. Distribution or reproduction of this work in whole or in part requires full attribution of the original publication, including its DOI. [DOI: 10.1117/1.JRS.8.083675]
\end{abstract}

Keywords: land surface temperature; emissivity; validation; moderate-resolution imaging spectrometer; advanced spaceborne thermal emission and reflection radiometer.

Paper 13241 received Jul. 3, 2013; revised manuscript received Jan. 2, 2014; accepted for publication Jan. 22, 2014; published online Feb. 18, 2014.

\section{Introduction}

Land surface emissivity (LSE), as an intrinsic property of natural materials, is primarily determined by the physical state and composition of the relevant materials. ${ }^{1,2}$ As an indicator of material composition, LSE can be used for terrestrial and planetary geological studies, bedrock mapping, resource exploration, and land cover characterization. ${ }^{3-7} \mathrm{LSE}$ is also a critical input for accurately determining the land surface temperature (LST) ${ }^{8,9}$ LSE and LST together determine the longwave radiation in the land-surface radiation and energy budgets and are the key input parameters in climatic, hydrological, ecological, and biogeochemical models. ${ }^{10-12}$

Regarding the algorithms developed for the retrieval of a certain particular biogeophysical parameters from satellite or aircraft data, the validation or testing by using field measurements is critical because the parameter accuracy determines its usefulness in applications. ${ }^{13-15}$ The feedback from the validation is also helpful to improve the designed algorithms. The moderateresolution imaging spectroradiometer (MODIS) onboard NASA's Earth Observation System satellite can provide moderate spatial resolution [5 km for Version 4 and 4.1 (V4 and 4.1),

*Address all correspondence to: Jie Cheng, E-mail: brucechan2003@126.com 
0.05 deg for Version 5 (V5)] LST and emissivity product twice daily, which has been used to map global broadband emissivity, ${ }^{16}$ estimate surface longwave radiation, ${ }^{17}$ and develop an emissivity database for the retrieval of atmospheric properties. ${ }^{18,19}$ Many studies have been conducted for the validation of the MODIS LST product. ${ }^{11,20,21}$ However, the validation of the MODIS LSE product is relatively scarce, especially the validation using field-measured emissivity. As a result, the MODIS emissivity product is not well recognized by the remote sensing community. Wang and Liang calculated the broadband emissivity using V4 and V5 of the MODIS monthly LST and LSE product V4 and V5 over six surface radiation budget network sites from 2000 to $2007 .{ }^{22}$ The broadband emissivity of the V5 data is greater than that of the V4 data at each site, and the seasonal variation of the V5 broadband emissivity data is very small. Hulley and Hook compared the V4, 4.1, and 5 of the MODIS emissivity products (MOD11B1) and validated them with the laboratory-measured emissivity of sand samples. ${ }^{23}$ The MOD11B1 V4.1 emissivity values most closely matched the laboratory results, with a mean absolute difference of 0.0065 , and the V5 emissivity was overestimated in all bands, with a mean absolute difference of 0.0193 , when compared to the laboratory results. Gottsche and Hulley validated six satellite-retrieved LSE products over land gravel plains and sand dunes in the hyper-arid Namib desert in Namibia using the in situ LSE obtained by the one-lid emissivity box method. ${ }^{24}$ The MOD11B1 V4.1 emissivity data frequently exhibited more extreme values (e.g., <0.92), and the MOD11B1 V5 emissivity data overestimated the actual values. The laboratory-measured emissivity data may differ from the field-measured emissivity as the surface microstructure is disturbed when the sand is transferred from the outdoors to the laboratory. The date of the field measurements is not exactly matched with the acquisition day of the examined emissivity product in the study of Gottsche and Hulley. In this study, we validate the MODIS emissivity product using synchronous field measured emissivity collected over the Taklimakan Desert.

\section{Emissivity Products}

\subsection{MODIS Emissivity Product}

The MODIS/Terra LST and LSE (MOD11B1) product is tile-based and gridded in the sinusoidal projection produced daily at $5 \mathrm{~km}$ (exactly $4.63 \mathrm{~km}$ ) spatial resolution for both V4 and V4.1 and at $6 \mathrm{~km}$ (exactly $5.56 \mathrm{~km}$ ) spatial resolution for V5. ${ }^{21}$ The MOD11B1 product is produced using the physically based day/night algorithm, which consists of 14 equations for the solution of 14 land-surface and atmospheric parameters (namely, the daytime and nighttime LSTs, the LSEs of the seven bands, the daytime and nighttime temperatures and water vapor contents of the lower layer of the atmosphere, and the bidirectional reflectance factor) based on the day/night observations of the seven infrared MODIS bands (bands 20, 22, 23, 29, 31, 32, and 33). ${ }^{25}$ The change in the spatial resolution from V4 to V5 was implemented to avoid resampling in the latitude direction when computing the MOD11C3 climate model grids. Another change from V4 to V5 is that the emissivity for the split-window (SW) algorithm ${ }^{26}$ is partly incorporated in the V4 product, i.e., it is used as an initial emissivity value for iteratively solving the constructed equations, while the SW emissivity is fully incorporated into the V5 product.

The MOD11B1 product tile H24V5 covers the location of the field measurements. The MOD11B1 product was reprojected from sinusoidal to geographical latitude/longitude to produce a spatial match. The bands $29(8.52 \mu \mathrm{m}), 31(11.02 \mu \mathrm{m})$, and $32(12.54 \mu \mathrm{m})$ of the MOD11B1 emissivity data were used for comparison. The quality of each pixel is guaranteed by examining the quality control information. Only pixels with good quality were considered. Figure 1 shows the MOD11B1 V4.1 emissivity data acquired on June 6, 2011, for band 29.

\subsection{Advanced Spaceborne Thermal Emission and Reflection Radiometer Temperature and Emissivity Products}

The advanced spaceborne thermal emission and reflection radiometer (ASTER) was launched on December 19, 1999, on NASA's Terra satellite. ASTER has five thermal infrared (TIR) bands in the spectral range of 8 to $12 \mu \mathrm{m}$ and a high spatial resolution of $90 \mathrm{~m}$. The ASTER temperature and 

products...

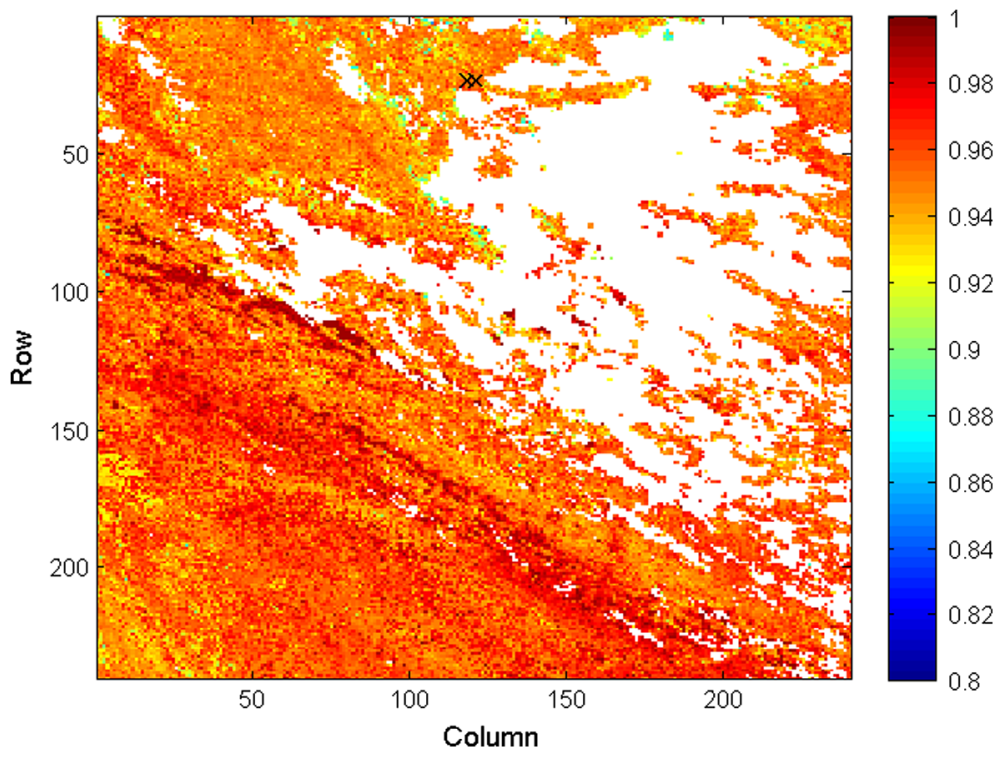

Fig. 1 MOD11B1 band 31 emissivity product (V4.1) acquired on June 6, 2011 (MOD11B1 tile H24V5). The black crosses represent the geographical locations of measurement sites.

emissivity separation (TES) algorithm was used to produce the LST and LSE products (ASTER standard data products AST05 and AST08). ${ }^{4}$ The TES algorithm is a hybrid algorithm that combines the advantages of the normalized emissivity method, ${ }^{27}$ the spectral ratio method, and the difference between the minimum emissivity and the maximum emissivity. ${ }^{28}$ The primary goal of the ASTER TES algorithm is to determine the emissivity for rocks and soils. ${ }^{4,29}$ The accuracy of emissivity measurements for rocks and soils is guaranteed. Field validation of the ASTER TES algorithm demonstrated that TES generally performed with nominal accuracy $(\sim \pm 1.5 \mathrm{~K}$ for temperature, and $\sim \pm 0.015$ for emissivity). ${ }^{30}$ Some other validation works also indicate that the ASTER TIR emissivity data achieved high accuracy over arid and semi-arid areas. ${ }^{30-33}$ Regarding vegetation, the accuracy of the emissivity inversion cannot meet the design goal due to either the low spectral contrast of the vegetation emissivity or the presence of high atmospheric temperatures and humidity, as reported by several authors. ${ }^{13,29,34}$ When the TES algorithm was modified multiple times to accommodate the low emissivity contrasts and error in the measured data, the accuracy was improved over the first version. ${ }^{35}$ Generally speaking, the ASTER data exhibit the highest spatial resolution $(90 \mathrm{~m})$ and the most accurate satellite emissivity product.

The ASTER temperature and emissivity products were used to characterize the homogeneity of the validation sites. We did not acquire the ASTER data on the date of the field measurements, but we did acquire two ASTER images on June 3 and June 10, 2011.

\section{Validation Data}

\subsection{Taklimakan Desert}

The Taklimakan Desert is the largest active desert in China and the second largest in the world, and it lies in a depression between two high, rugged mountain ranges. The Taklimakan Desert is located at $72^{\circ} 14^{\prime}$ to $90^{\circ} 37^{\prime} \mathrm{E}, 36^{\circ} 23^{\prime}$ to $42^{\circ} 6^{\prime} \mathrm{N}$. The middle of the desert has a typical continental climate, with high winds and temperatures as well as low annual rainfall. In the sand dune areas of the Taklimakan Desert, the dune height is generally 100 to $200 \mathrm{~m}$, and it occasionally can reach up to $300 \mathrm{~m}$. The types of sand dunes in the Taklimakan Desert are compound, sand hills, sand ridges, and tower-type dunes with honeycomb-like, feathery, scaly, and unpredictable surface types. During sand storms and dusty weather, the grain size parameter of Taklimakan Desert ranges from 3 to $4(0.0625$ to $0.125 \mathrm{~mm})$, with a standard deviation of 0.03 to $0.98 .{ }^{36}$ During the day in the Taklimakan Desert, the scorching sun can heat the dazzling silver sand to surface temperatures that can reach 70 to $80^{\circ} \mathrm{C}$ and strong 

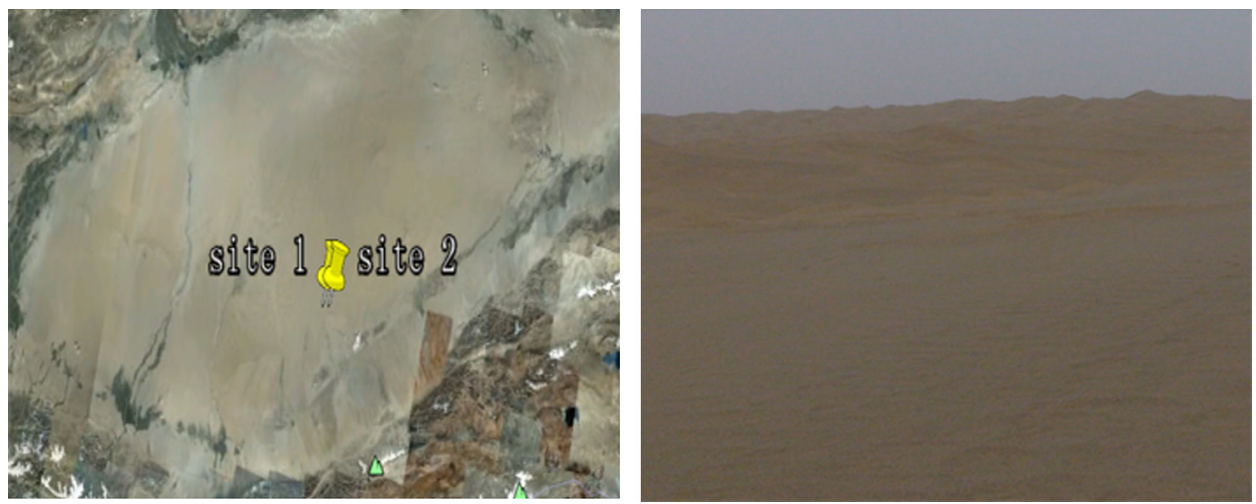

Fig. 2 Geographical locations and the landscape of the validation sites.

evaporation occurs. Figure 2 presents the geographical locations and the landscape of the validation sites.

\subsection{Field Measurements}

We conducted the field measurements on June 6, 2011, at the Taklimakan Desert. In total, two flat and homogeneous sand sites in the central area of the Taklimakan sand were selected. The geographical locations of the sites are $\left(38.97^{\circ} \mathrm{N}, 83.57^{\circ} \mathrm{E}\right)$ for site 1 and $\left(38.97^{\circ} \mathrm{N}, 83.69^{\circ} \mathrm{E}\right)$ for site 2 . The Model 102 Portable FT-IR Spectrometer (Model 102F) and a Labsphere gold plate were used to measure spectral radiance emitted by the target and by the environment under a clear sky. The spectral range of the Model $102 \mathrm{~F}$ instrument is 2 to $16 \mu \mathrm{m}$, and the spectral resolution can be chosen to be 4,8 , or $16 \mathrm{~cm}^{-1}$, with an optional resolution of $2 \mathrm{~cm}^{-1}$. The operating temperature range is 15 to $35^{\circ} \mathrm{C}$. Before the measurement, the Model $102 \mathrm{~F}$ was preheated until the internal temperature was steady. Next, the Model $102 \mathrm{~F}$ was calibrated by a thermal infrared blackbody at five known temperatures. The target and the infrared golden plate were measured alternatively. Note that the field measurement occurred only under the weather conditions of no clouds, no near-surface winds, and a relatively stable atmosphere. The emissivity spectrum was derived from the radiometric measurements by the iterative spectrally smooth temperature and emissivity separation algorithm. ${ }^{37}$ For each site, we conducted three measurements, and then we randomly chose two points within a distance of $\sim 500 \mathrm{~m}$ from the site and conducted three measurements at each of the points. The derived nine emissivity spectra were averaged and regarded as the emissivity of the site. Figure 3 shows the field-measured mean emissivity spectra of site 1 and site 2 in

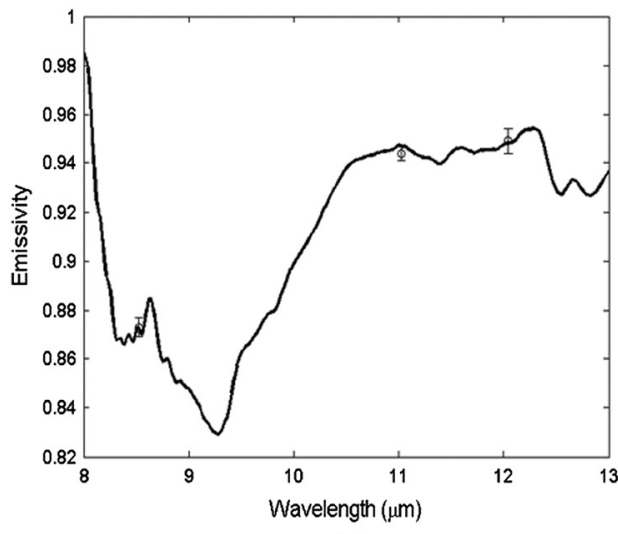

(a)

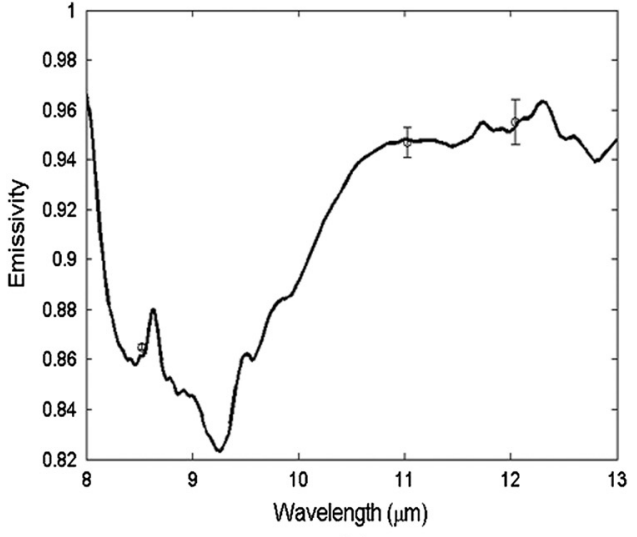

(b)

Fig. 3 Field measured mean emissivity spectra of site 1 (a) and site 2 (b), and the mean emissivity and standard deviation of the respective sites convolved with the moderate-resolution imaging spectroradiometer spectral response functions for bands 29, 31, and 32 . 
the spectral range of 8 to $13 \mu \mathrm{m}$, and the average and standard deviation of the emissivity corresponding to the MODIS bands 29, 31, and 32 .

\section{Results and Discussion}

The homogeneity of the validation site is a prerequisite for the validation of the satellite products. The ASTER temperature and emissivity data were used to characterize the homogeneity of the validation sites. Figure 4 shows the emissivity for ASTER bands 10 and 14. Visually, site 1 is more homogeneous than site 2 .

According to Norman and Becker ${ }^{38}$ there are two types of emissivity for each nonisothermal pixel, i.e., $r$-emissivity and $e$-emissivity. Centered on the geographical locations of the validation sites, we calculated $r$-emissivity and $e$-emissivity for the subregions of $62 * 62,124 * 124,248 *$ 248 ASTER pixels ( $\sim 1 * 1,2 * 2,4 * 4$ MODIS pixels) using Eqs. (1) and (2). The $r$-emissivity can be used to express the area weighting of the component emissivity, which has no relationship with the component temperature. If a pixel has $N$ components, then

$$
\varepsilon_{r, i}(\theta, \phi)=\sum_{k=1}^{N} a_{k} \varepsilon_{r, i, k}(\theta, \phi),
$$

where $i$ represents the band, $a_{k}$ is the normalized-area proportion of component $k$, and $\varepsilon_{r, i, k}(\theta, \phi)$ is the emissivity of each component in the direction of $(\theta, \phi)$. The $e$-emissivity is defined as the ratio of the total radiation of a natural object surface to the blackbody radiation with an identical temperature distribution. When the pixel has $N$ components,

$$
\varepsilon_{e, i}(\theta, \phi)=\frac{\sum_{k=1}^{N} a_{k} \varepsilon_{r, i, k}(\theta, \phi) T_{R, i, k}^{n}(\theta, \phi)}{\sum_{k=1}^{N} a_{k} T_{R, i, k}^{n}(\theta, \phi)},
$$

where $T_{R, j, k}^{n}(\theta, \phi)$ is the approximation to the exponential function of blackbody radiation. Thus, the $e$-emissivity is a function of an object's component temperatures. Figures 5 and 6 show the calculated $r$-emissivity and $e$-emissivity using ASTER TIR emissivity on June 3 and June 10, 2011, for the three subregions. For site 1, the absolute difference between $r$-emissivity and $e$ emissivity for each TIR band is $<0.0007$ on June 3, 2011, and the value of the difference is $<0.00002$ on June 10, 2011. The corresponding difference values for site 2 on June 3 and June 10, 2011, are 0.0005 and 0.0009 , respectively. Thus, the definition of emissivity will not affect the validation results in this study. Hereafter, we will use $r$-emissivity in the comparison. Because the size (area ratio) of each ASTER pixel within the subregion is the same, the $r$-emissivity equals the average emissivity. We also calculated the standard deviation of the ASTER emissivity for each subregion.

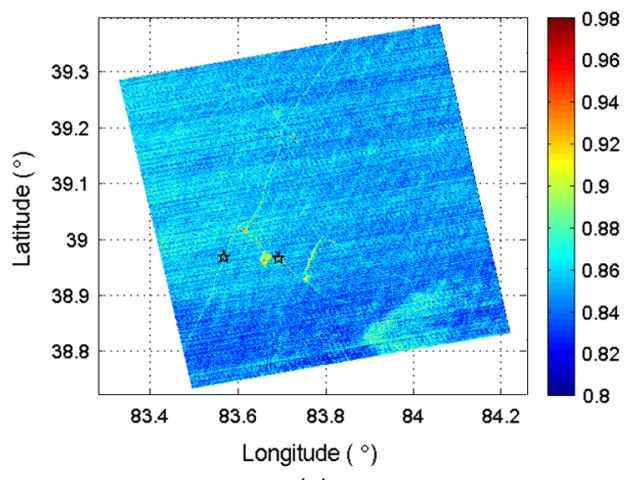

(a)

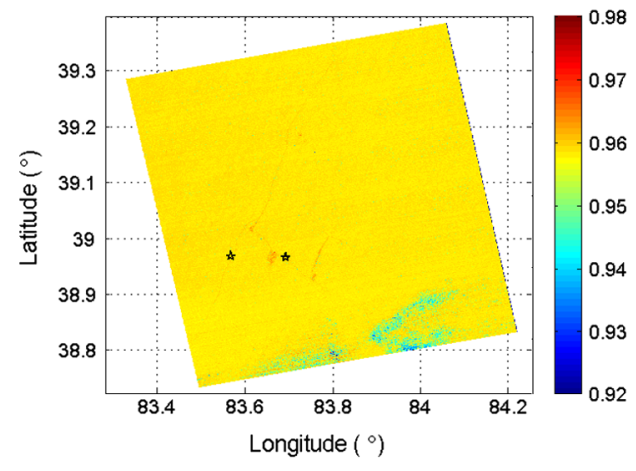

(b)

Fig. 4 Emissivity data in the advanced spaceborne thermal emission and reflection radiometer bands 10 (a) and 14 (b) acquired on June 10, 2011. The black pentagrams represent the geographical locations of the measurement sites. 

products...
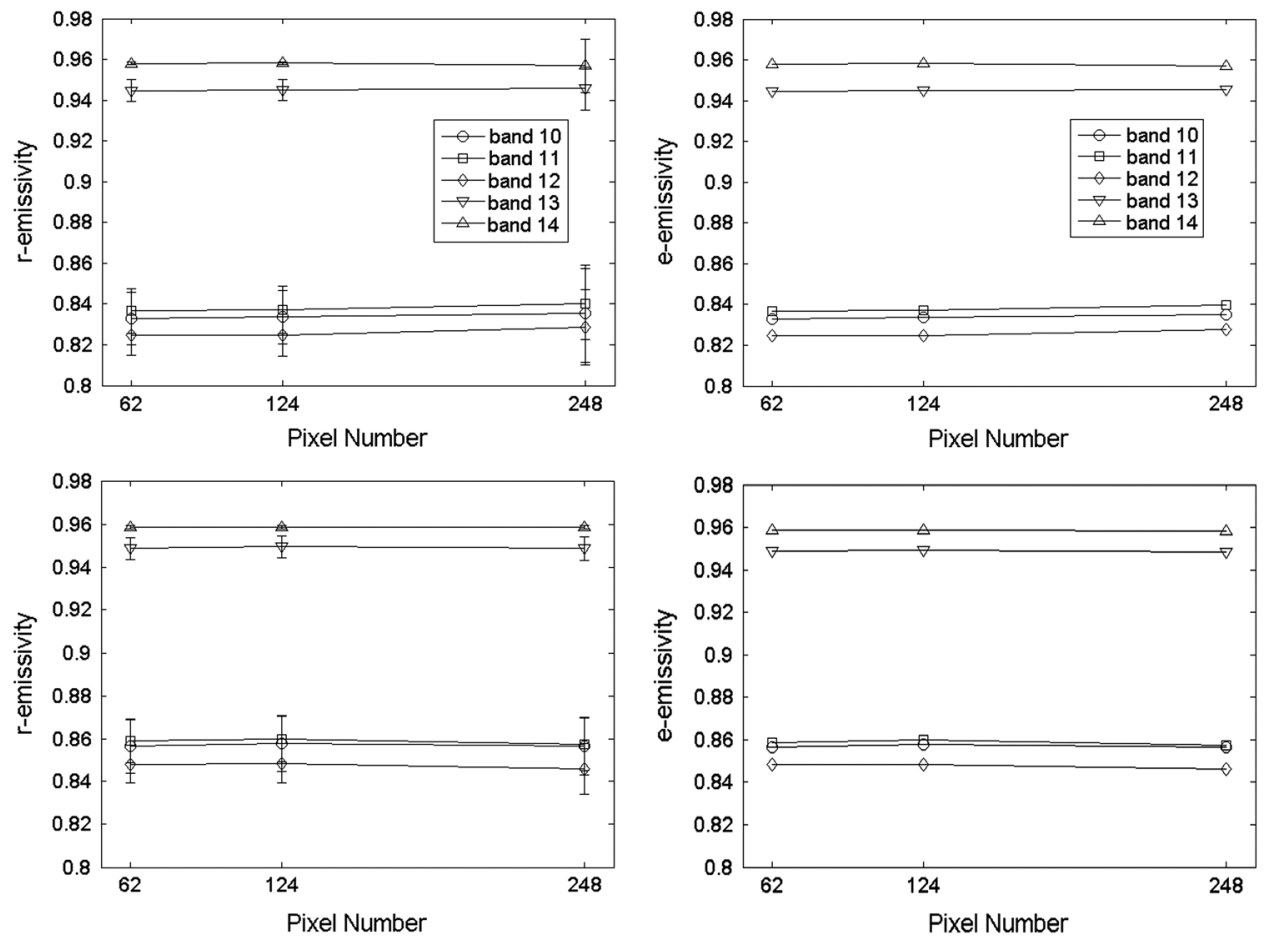

Fig. 5 The $r$-emissivity and e-emissivity derived from the AST05 and AST08 on June 3, 2011 (upper), and June 10, 2011 (lower), for site 1.

The emissivity variation of site 1 is slightly smaller than that of site 2 as site 1 is relatively more homogeneous than site 2 (Fig. 4). When the subregion changes from $64 * 64$ to $128 * 128$, the variation of the average emissivity variation is $<0.001$ and 0.002 for sites 1 and 2 , respectively, and the variation of the emissivity standard deviation variation is $<0.002$ and 0.002 for sites 1 and 2, respectively. The variation of the average emissivity is $<0.003$ and 0.006 for sites 1
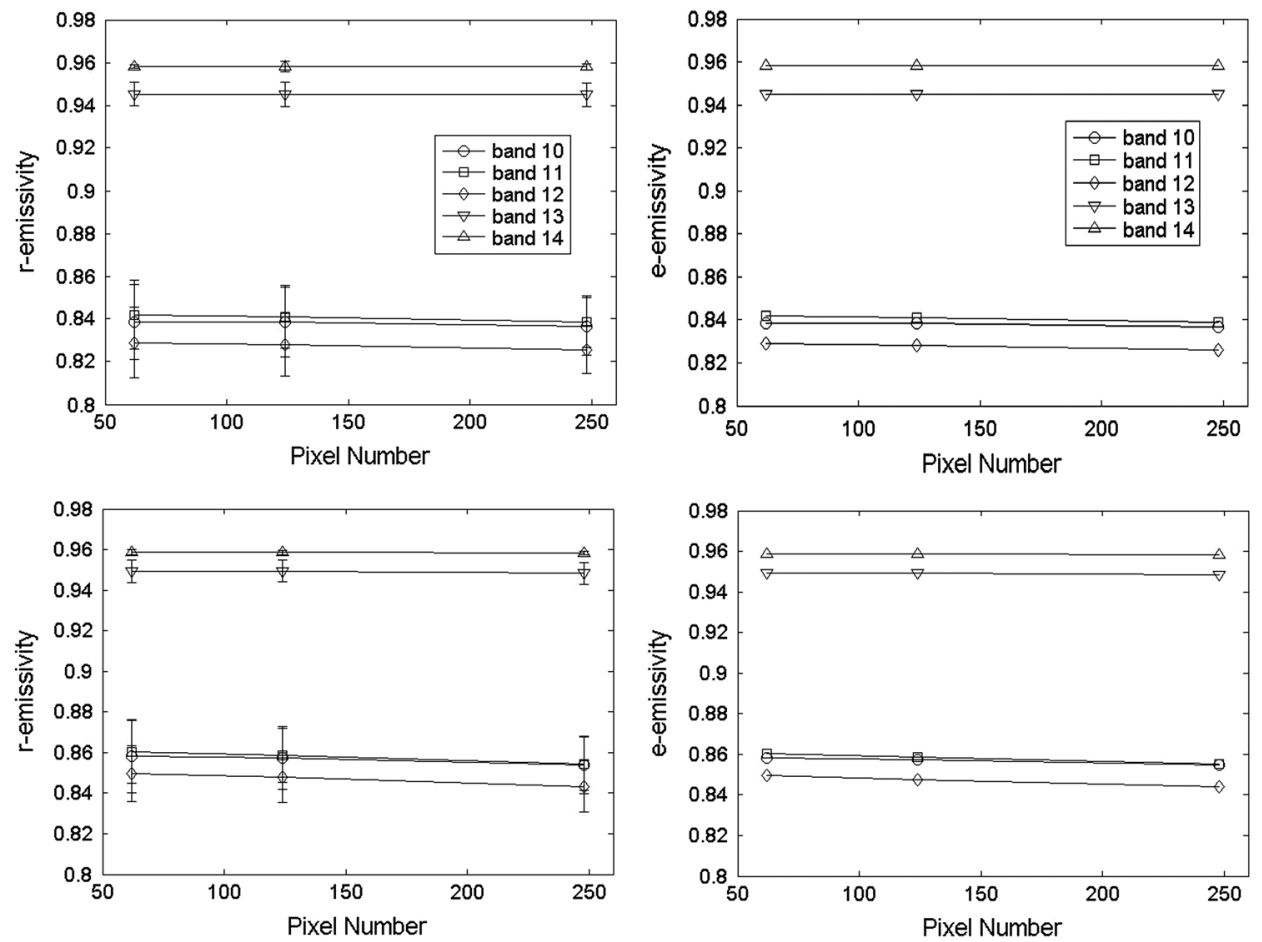

Fig. 6 The $r$-emissivity and e-emissivity derived from the AST05 and AST08 on June 3, 2011 (upper), and June 10, 2011 (lower), for site 2. 

products...
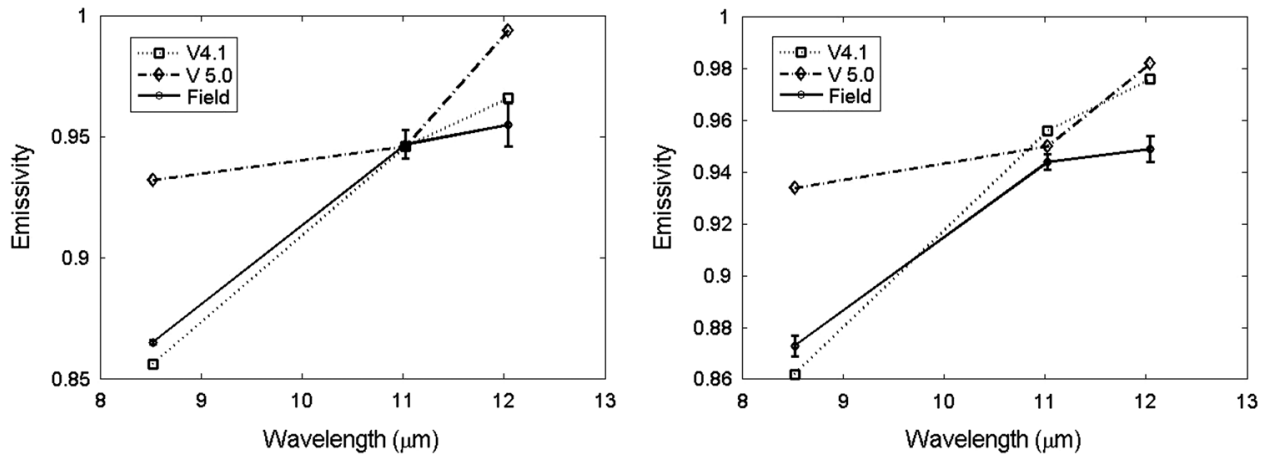

Fig. 7 Comparison of the MOD11B1 emissivity data with the field-measured emissivity data. Error bars denote the standard deviation of field-measured emissivity.

and 2, respectively, and the variation of emissivity standard deviation is $<0.004$ and 0.005 for sites 1 and 2, respectively, as the subregion changes from $64 * 64$ to $254 * 254$. The emissivity standard deviation decreases gradually from band 10 to band 14 for each subregion, i.e., band 10 exhibits the largest standard deviation, while band 14 exhibits the smallest standard deviation. The emissivity standard deviation for bands 10 to 12 ranges from 0.012 to 0.018 , and the corresponding value for bands 13 and 14 ranges from 0.001 to 0.006 . The emissivity standard deviation tends to decrease when the subregion size increases for bands 10 to 12 . The variation of the average emissivity is quite small, and the larger emissivity standard deviation is on the order of the ASTER nominal emissivity accuracy with respect to the increasing size of subregions. Therefore, the measurement sites can be considered to be homogeneous.

We extracted the emissivity from MOD11B1 for bands 29, 31, and 32, and compared the results to the emissivity calculated by convolving the field-measured emissivity spectra with the filter function of MODIS bands 29, 31, and 32. The comparison results are shown in Fig. 7 and Table 1. Generally, the MOD11B1 V4.1 emissivity agrees better with the field measurements than does the MOD11B1 V5 emissivity. The MOD11B1 V5 emissivity overestimates the sand emissivity for all three bands. This overestimation is consistent with the results of Hulley and Hook. ${ }^{23}$ The SW emissivity is derived using a land-cover classification method and cannot capture the actual emissivity variation of the land surface, especially the sand. Thus, the full incorporation of SW emissivity with the day/night algorithm in V5 degrades the accuracy of the retrieved emissivity over the desert. The MOD11B1 V4.1 product underestimates the band 29

Table 1 The difference between MOD11B1 V4.1 and V5 emissivity data and the field measurements.

\begin{tabular}{|c|c|c|c|c|c|c|c|}
\hline \multirow[b]{2}{*}{ Version } & \multirow[b]{2}{*}{ Band } & \multicolumn{3}{|c|}{ Site 1} & \multicolumn{3}{|c|}{ Site 2} \\
\hline & & MODIS & Field & Diff. & MODIS & Field & Diff. \\
\hline \multirow[t]{3}{*}{ V4.1 } & 29 & 0.862 & 0.873 & -0.011 & 0.856 & 0.865 & -0.009 \\
\hline & 31 & 0.956 & 0.944 & 0.012 & 0.946 & 0.947 & -0.001 \\
\hline & 32 & 0.976 & 0.949 & 0.027 & 0.966 & 0.955 & 0.011 \\
\hline \multicolumn{2}{|c|}{ Mean absolute diff. } & & 0.017 & & & 0.007 & \\
\hline \multirow[t]{3}{*}{ V5 } & 29 & 0.932 & 0.873 & 0.059 & 0.932 & 0.865 & 0.067 \\
\hline & 31 & 0.964 & 0.944 & 0.020 & 0.964 & 0.947 & 0.017 \\
\hline & 32 & 0.972 & 0.949 & 0.023 & 0.972 & 0.955 & 0.017 \\
\hline \multicolumn{2}{|c|}{ Mean absolute diff. } & & 0.034 & & & 0.037 & \\
\hline
\end{tabular}

Note: MODIS, moderate-resolution imaging spectrometer. 
emissivity with the bias of -0.011 and -0.009 for site 1 and site 2 , respectively, whereas the MOD11B1 V5 product overestimates the sand emissivity with the bias of 0.059 and 0.067 for site 1 and site 2, respectively. For band 31, both the MOD11B1 V4.1 and V5.0 products agree well with the measurements. The difference for MOD11B1 V4.1 and V5.0 is only 0.012 and 0.020 for site 1 . The bias becomes -0.001 and 0.017 for MOD11B1 V4.1 and V5.0, respectively, for site 2. Regarding band 32, both the MOD11B1 V4.1 and V5 overestimate the sand emissivity. The bias of MOD11B1 V4 is 0.027 and 0.011 for site 1 and site 2, respectively, and the bias of MOD11B1 V5 is 0.023 and 0.017 for site 1 and site 2, respectively. The bias of MOD11B1 V5 is quite large for band 29, and the emissivity of band 29 should not be used in real applications. Statistically, the average absolute bias of MOD11B1 V4.1 is 0.017 and 0.007 for site 1 and site 2, respectively, whereas the average bias of MOD11B1 V5 is 0.034 and 0.033 for site 1 and site 2, respectively. Evidently, the user must pay attention to the version when using MODIS emissivity product, especially the time series analysis using the MODIS emissivity product.

\section{Conclusion}

A field measurement was conducted over the central part of the Taklimakan Desert for the validation of the MODIS land surface emissivity products (MOD11B1) V4.1 and V5. The ASTER LST and LSE data acquired closely before and after the overpass of MODIS was used to characterize the homogeneity of the measurement sites. Two types of emissivity ( $r$-emissivity and $e$-emissivity) were calculated for the subregions centered on the validation sites with sizes of $62 * 62,124 * 124$, and $248 * 248$ ASTER pixels. The absolute difference between $r$-emissivity and $e$-emissivity for each TIR band over the two validation sites is $<0.0009$. Thus, the definition of emissivity for a nonisothermal pixel will not influence the validation results. The variation of the average emissivity is quite small, and the largest emissivity standard deviation is on the order of the ASTER nominal emissivity accuracy with respect to the increasing size of the subregions. Thus, the validation sites can be thought of as homogeneous.

MOD11B1 V4.1 and V5 emissivity for bands 29,31, and 32 were compared to the emissivity calculated by convolving the field-measured emissivity spectra with the filter function of the MODIS bands 29, 31, and 32. The comparison results indicated that the V4.1 emissivity data agree well with the field measurements, with mean absolute difference of 0.007 and 0.017 for site 1 and site 2, respectively. The mean absolute differences of V5 emissivity versus the field measurements were 0.036 and 0.033 for site 1 and site 2, respectively. Thus, the version must be considered when MOD11B1 is used in real applications, especially for the time series analysis.

\section{Acknowledgments}

The moderate-resolution imaging spectroradiometer and advanced spaceborne thermal emission and reflection radiometer data are obtained from http://reverb.echo.nasa.gov/reverb. This work was supported by the National Natural Science Foundation of China via grant 41371323, the National High Technology Research and Development Program of China via grant 2013AA121201, and Beijing Higher Education Young Elite Teacher project via Grant YETP0233.

\section{References}

1. Z.-L. Li et al., "Land surface emissivity retrieval from satellite data," Int. J. Remote Sens. 34(9-10), 1-44 (2013).

2. J. Cheng et al., "Temperature and emissivity separation from ground-based MIR hyperspectral data," IEEE Trans. Geosci. Remote Sens. 49(4), 1473-1484 (2011), http://dx.doi.org/10 $.1109 /$ TGRS.2010.2076818.

3. A. N. French and A. Inamdar, "Land cover characterization for hydrological modelling using thermal infrared emissivities," Int. J. Remote Sens. 31(14), 3867-3883 (2010), http://dx.doi.org/10.1080/01431161.2010.483491.

4. A. R. Gillespie et al., "A temperature and emissivity separation algorithm for advanced spaceborne thermal emission and reflection radiometer (ASTER) images," IEEE Trans. Geosci. Remote Sens. 36(4), 1113-1126 (1998), http://dx.doi.org/10.1109/36.700995. 
5. R. G. Vaughan, W. M. Calvin, and J. V. Taranik, "SEBASS hyperspectral thermal infrared data: surface emissivity measurement and mineral mapping," Remote Sens. Environ. 85(1), 48-63 (2003), http://dx.doi.org/10.1016/S0034-4257(02)00186-4.

6. L. Kirkland et al., "First use of airborne thermal infrared hyperspectral scanner for compositional mapping," Remote Sens. Environ. 80(3), 447-459 (2002), http://dx.doi.org/10.1016/ S0034-4257(01)00323-6.

7. S. J. Hook et al., "A comparison of techniques for extracting emissivity information from thermal infrared data for geological studies," Remote Sens. Environ. 42(2), 123-135 (1992), http://dx.doi.org/10.1016/0034-4257(92)90096-3.

8. P. Dash et al., "Land surface temperature and emissivity estimation from passive sensor data: theory and practice-current trends," Int. J. Remote Sens. 23(13), 2563-2594 (2002), http://dx.doi.org/10.1080/01431160110115041.

9. S. Liang, "An optimization algorithm for separating land surface temperature and emissivity from multispectral thermal infrared imagery," IEEE Trans. Geosci. Remote Sens. 39(2), 264-274 (2001), http://dx.doi.org/10.1109/36.905234.

10. S. Liang et al., "Review of estimation of land surface radiation and energy budgets from ground measurements, remote sensing and model simulation," IEEE J. Sel. Topics Earth Obs. Remote Sens. 3(3), 225-240 (2010), http://dx.doi.org/10.1109/JSTARS.2010.2048556.

11. F. Jacob et al., "Comparison of land surface emissivity and radiometric temperature derived from MODIS and ASTER sensors," Remote Sens. Environ. 90(2), 137-152 (2004), http://dx .doi.org/10.1016/j.rse.2003.11.015.

12. T. Schmugge et al., "Temperature and emissivity separation from multispectral thermal infrared observations," Remote Sens. Environ. 79(2-3), 189-198 (2002), http://dx.doi .org/10.1016/S0034-4257(01)00272-3.

13. J. C. Jimenez-Munoz et al., "Improved land surface emissivities over agricultural areas using ASTER NDVI," Remote Sens. Environ. 103(4), 474-487 (2006), http://dx.doi.org/ 10.1016/j.rse.2006.04.012.

14. Y. Yu et al., "Validation of GOES-R satellite land surface temperature algorithm using SURFRAD ground measurements and statistical estimates of error properties," IEEE Trans. Geosci. Remote Sens. 50(3), 704-713 (2012), http://dx.doi.org/10.1109/TGRS.2011.2162338.

15. S. Liang et al., "Validating MODIS land surface reflectance and albedo products: methods and preliminary results," Remote Sens. Environ. 83(2), 149-162 (2002), http://dx.doi.org/10 .1016/S0034-4257(02)00092-5.

16. K. Ogawa, T. Schmugge, and S. Rokugawa, "Estimating broadband emissivity of arid regions and its seasonal variations using thermal infrared remote sensing," IEEE Trans. Geosci. Remote Sens. 46(2), 334-343 (2008), http://dx.doi.org/10.1109/TGRS.2007 .913213.

17. W. Wang, S. Liang, and J. A. Augustine, "Estimating high spatial resolution clear-sky land surface upwelling longwave radiation from MODIS data," IEEE Trans. Geosci. Remote Sens. 47(5), 1559-1570 (2009), http://dx.doi.org/10.1109/TGRS.2008.2005206.

18. S. W. Seemann et al., "Development of a global infrared land surface emissivity database for application to clear sky sounding retrieval from multispectral satellite radiance measurements," J. Appl. Meteorol. Climatol. 47(1), 108-123 (2008), http://dx.doi.org/10.1175/ 2007JAMC1590.1.

19. V. Capelle et al., "Infrared continental surface emissivity spectra and skin temperature retrieved from IASI observations over the tropics," J. Appl. Meteorol. Climatol. 51(6), 1164-1179 (2012), http://dx.doi.org/10.1175/JAMC-D-11-0145.1.

20. Z. Wan et al., "Validation of the land surface temperature products retrieved from terra moderate resolution imaging sepctrometer data," Remote Sens. Environ. 83(1-2), 163180 (2002), http://dx.doi.org/10.1016/S0034-4257(02)00093-7.

21. Z. Wan, "New refinements and validation of MODIS land-surface temperature/emissivity products," Remote Sens. Environ. 112(1), 59-74 (2008), http://dx.doi.org/10.1016/j.rse.2006.06.026.

22. K. Wang and S. Liang, "Evaluation of ASTER and MODIS land surface temperature and emissivity products using long-term surface longwave radiation observations at SURFRAD sites," Remote Sens. Environ. 113(7), 1556-1565 (2009), http://dx.doi.org/10.1016/j.rse .2009.03.009. 
23. G. C. Hulley and S. J. Hook, "Intercomparison of versions 4, 4.1 and 5 of the MODIS land surface temperature and emissivity products and validation with laboratory measurements of sand samples from the Namib desert, Namibia," Remote Sens. Environ. 113(6), 13131318 (2009), http://dx.doi.org/10.1016/j.rse.2009.02.018.

24. F.-M. Gottsche and G. C. Hulley, "Validation of six satellite-retrieved land surface emissivity products over two land cover types in a hyper-arid region," Remote Sens. Environ. 124, 149-158 (2012), http://dx.doi.org/10.1016/j.rse.2012.05.010.

25. Z. Wan and Z.-L. Li, "A physics-based algorithm for retrieving land-surface emissivity and temperature from EOS/MODIS data," IEEE Trans. Geosci. Remote Sens. 35(4), 980-996 (1997), http://dx.doi.org/10.1109/36.602541.

26. Z. Wan and J. Dozier, "A generalized split-window algorithm for retrieving land-surface temperature form space," IEEE Trans. Geosci. Remote Sens. 34(4), 892-905 (1996), http://dx.doi.org/10.1109/36.508406.

27. A. R. Gillespie, "Lithologic mapping of silicate rocks using TIMS," in Proc. TIMS Data Users' Workshop, pp. 29-44, NASA, USA (1985).

28. T. Matsunaga, "A temperature-emissivity separation method using an empirical relationship between the mean, the maximum, the minimum of the thermal infrared emissivity spectrum,” J. Remote Sens. Soc. Jpn. 14(3), 230-241 (1994).

29. A. R. Gillespie et al., "Residual errors in ASTER temperature and emissivity products AST08 and AST05," Remote Sens. Environ. 115(12), 3681-3694 (2011), http://dx.doi .org/10.1016/j.rse.2011.09.007.

30. D. E. Sabol et al., "Field validation of the ASTER temperature-emissivity separation algorithm," Remote Sens. Environ. 113(11), 2328-2344 (2009), http://dx.doi.org/10.1016/j.rse 2009.06.008.

31. G. C. Hulley, S. J. Hook, and A. M. Baldridge, "Validation of the North American ASTER land surface emissivity database (NAALSED) version 2.0 using pseudo-invariant sand dune sites,” Remote Sens. Environ. 113(10), 2224-2233 (2009), http://dx.doi.org/10.1016/j.rse 2009.06.005.

32. M. Mira et al., "Analysis of ASTER emissivity product over an arid area in southern New Mexico, USA," IEEE Trans. Geosci. Remote Sens. 49(4), 1316-1324 (2011), http://dx.doi .org/10.1109/TGRS.2010.2061858.

33. T. Matsunaga et al., "Early evaluation of ASTER emissivity products and its application to environmental and geologic studies," Proc. SPIE 4486, 20-30 (2001), http://dx.doi.org/10 $.1117 / 12.455121$.

34. J. C. Jimenez-Munoz et al., "Improved land surface emissivities over agricultural areas using ASTER NDVI,” Remote Sens. Environ. 103(4), 474-487 (2006), http://dx.doi.org/ 10.1016/j.rse.2006.04.012.

35. W. T. Gustafson, A. R. Gillespie, and G. J. Yamada, "Revisions to the ASTER temperature/ emissivity separation algorithm," in Second Recent Advances in Quantitative Remote Sensing, J. A. Sobrino, Ed., pp. 770-775, Publishion de la Universitat de Valencia, Spain (2006).

36. W. Huo et al., "The research on grain size characteristics of desert in North of China," Res. Soil Water Conserv. 18(6), 11-16 (2011).

37. C. C. Borel, "Surface emissivity and temperature retrieval for a hyperspectral sensor," in Proc. IEEE Conf. on Geoscience and Remote Sensing, Seattle, WA, pp. 546-549 (1998).

38. J. M. Norman and F. Becker, "Terminology in thermal infrared remote sensing of natural surfaces," Remote Sens. Rev. 12(3-4), 159-173 (1995), http://dx.doi.org/10.1080/ 02757259509532284 .

Jie Cheng is an associate professor at Beijing Normal University. He received his $\mathrm{PhD}$ degree in cartography and remote sensing from the Institute of Remote Sensing Applications of Chinese Academy of Sciences, Beijing, China, in 2008. His main research interests focus on estimation of land surface variables from satellite observations, studies on surface energy balance, and radiative transfer modeling.

Biographies of the other authors are not available. 